\title{
Transition probabilities in Gd III
}

\author{
E. Biémont ${ }^{1,2}$, G. Kohnen ${ }^{1}$, and P. Quinet ${ }^{1,2}$ \\ 1 Astrophysique et Spectroscopie, Université de Mons-Hainaut, 7000 Mons, Belgium \\ 2 IPNAS (Bât. B15), Université de Liège, Sart Tilman, 4000 Liège, Belgium \\ Received 21 June 2002 / Accepted 10 July 2002

\begin{abstract}
Theoretical lifetimes, calculated with inclusion of core-polarization effects, have been determined for five $4 f^{7} 6 p$ levels of doubly ionized gadolinium. They agree quite well with recent experimental values measured by time-resolved laserinduced fluorescence spectroscopy. From this agreement, the accuracy of a first set of Gd III transition probabilities, calculated for $4 f^{7} 5 d-4 f^{7} 6 p$ and $4 f^{7} 6 s-4 f^{7} 6 p$ transitions of astrophysical interest, has been assessed.
\end{abstract}

Key words. atomic data - stars: chemically peculiar

\section{Introduction}

Singly ionized gadolinium is well represented in the solar spectrum and its abundance is, according to the latest determinations, in good agreement with the meteoritic value (Bergström et al. 1988; Anders \& Grevesse 1989). Gd II has been also identified in many other stars of different types: as a few examples, let us mention the $\mathrm{Hg}-\mathrm{Mn}$ stars (Adelman 1989), the Ap stars HR465 and HR7575 (Hartoog et al. 1973), the Mira variables (Grudzinska 1985), the Ba stars (Lambert 1985) or the Am star 32 Aqr (Magazzu \& Cowley 1986). Strong lines of lanthanides, including Gd II, have been observed in the spectrum of a cool rapidly rotating Ap star (roAp) i.e. the Przybylski's star (HD101065, V816 Cen) (Cowley et al. 2000). Gd II lines have also been analyzed in the K giant star CS 22892-052 by Sneden et al. (1996) who found an abundance result corresponding to a scaled solar system $r$-process value.

In general, the abundances of the lanthanide rare-earths (RE), deduced in magnetic Ap stars and in metallic line A stars, exceed considerably the solar system values and are generally larger, by about one order of magnitude, in the first than in the second group. In many magnetic Ap stars however, the effective temperatures imply that most of the atoms should be doubly ionized. Until recently, abundance values in such stars were mostly obtained from the non-dominant ionization stage (i.e. the second spectrum) due to the fact that transition probabilities for doubly ionized RE elements were unavailable or that the strongest lines of the third spectra fall outside of the accessible spectral region (Cowley 1980). The situation has now changed and there has been, in recent years, a revival of interest for the doubly ionized species of the lanthanides. In particular, we have started a systematic investigation of the line intensities in these spectra

Send offprint requests to: $\mathrm{E}$. Biémont or P. Quinet e-mail: E.Biemont@ulg.ac.be or quinet@umh.ac.be within the framework of the DREAM project (for more details, see http://www.umh.ac.be/ astro/dream.shtml) and extensive tables of transition probabilities, tested in many cases by comparison with laser lifetime measurements, have been obtained for most of the doubly ionized elements of the group (Biémont et al. 1999, 2001a,b,c,d, 2002; Fedchak et al. 2000; Li et al. 2001; Palmeri et al. 2000; Zhang et al. 2001a, 2002a,b,c). The case of Gd III has not been considered so far and, for this reason, the first results are presented in the present paper.

\section{The spectrum of Gd III}

Gadolinium $(Z=64)$ has seven isotopes $(152,154,155,156$, 157,158 and 160) of which Gd 155, 156, 157, 158 and 160 are represented, in the solar system, by nearly equal amounts $(15,20,16,25$ and $22 \%$, respectively). These isotopes are essentially produced by the $r$-process, small amounts of the first four isotopes being due to the s-process.

Among the third RE spectra, doubly ionized gadolinium has the most complex electronic structure. This is due to the fact that the lowest configurations of this ion are characterized by a half-filled $4 \mathrm{f}$ subshell with the presence of an additional valence electron. As a result of this complexity, Gd III has been the subject of very few investigations in the past although lines of this ion have been observed in some astrophysical spectra such as those of the stars $\alpha^{2} C V n$ (Cohen et al. 1969) and HR465 (Bidelman et al. 1995). A search of the third spectra of RE, including Gd III, in IUE spectra of chemically peculiar stars has also been reported by Cowley \& Greenberg (1988).

According to the NIST compilation (Martin et al. 1978), only 25 energy levels belonging to the $4 \mathrm{f}^{8}, 4 \mathrm{f}^{7}\left({ }^{8} \mathrm{~S}^{\circ}\right) 5 \mathrm{~d}$, $4 \mathrm{f}^{7}\left({ }^{8} \mathrm{~S}^{\circ}\right) 6 \mathrm{~s}, 4 \mathrm{f}^{7}\left({ }^{8} \mathrm{~S}^{\circ}\right) 6 \mathrm{p}$ and $4 \mathrm{f}^{7}\left({ }^{8} \mathrm{~S}^{\circ}\right) 7 \mathrm{~s}$ configurations have been determined experimentally. These levels were deduced from 
Table 1. Adopted parameters for HFR calculations in Gd III.

\begin{tabular}{|c|c|c|c|c|}
\hline Config. & Parameter & $\begin{array}{r}\text { Ab initio } \\
\left(\mathrm{cm}^{-1}\right)\end{array}$ & $\begin{array}{r}\text { Adopted } \\
\left(\mathrm{cm}^{-1}\right)\end{array}$ & Ratio \\
\hline \multicolumn{5}{|l|}{ Even parity } \\
\hline \multirow[t]{11}{*}{$4 f^{7} 5 d$} & $E_{\mathrm{av}}$ & 97683 & 96777 & \\
\hline & $F^{2}(4 \mathrm{f}, 4 \mathrm{f})$ & 116091 & 98677 & $0.850 f$ \\
\hline & $F^{4}(4 \mathrm{f}, 4 \mathrm{f})$ & 72815 & 61893 & $0.850 f$ \\
\hline & $F^{6}(4 \mathrm{f}, 4 \mathrm{f})$ & 52379 & 44522 & $0.850 f$ \\
\hline & $\zeta_{4 \mathrm{f}}$ & 1582 & 1582 & $1.000 f$ \\
\hline & $\zeta_{5 \mathrm{~d}}$ & 1034 & 957 & 0.926 \\
\hline & $F^{2}(4 \mathrm{f}, 5 \mathrm{~d})$ & 25640 & 21794 & $0.850 f$ \\
\hline & $F^{4}(4 \mathrm{f}, 5 \mathrm{~d})$ & 12246 & 10409 & $0.850 f$ \\
\hline & $G^{1}(4 \mathrm{f}, 5 \mathrm{~d})$ & 11662 & 8227 & $0.705 *$ \\
\hline & $G^{3}(4 \mathrm{f}, 5 \mathrm{~d})$ & 9358 & 6601 & $0.705 *$ \\
\hline & $G^{5}(4 \mathrm{f}, 5 \mathrm{~d})$ & 7105 & 5011 & $0.705 *$ \\
\hline \multirow[t]{6}{*}{$4 f^{7} 6 s$} & $E_{\mathrm{av}}$ & 104059 & 102080 & \\
\hline & $F^{2}(4 \mathrm{f}, 4 \mathrm{f})$ & 116684 & 99182 & $0.850 f$ \\
\hline & $F^{4}(4 \mathrm{f}, 4 \mathrm{f})$ & 73216 & 62234 & $0.850 f$ \\
\hline & $F^{6}(4 \mathrm{f}, 4 \mathrm{f})$ & 52675 & 44774 & $0.850 f$ \\
\hline & $\zeta_{4 \mathrm{f}}$ & 1588 & 1588 & $1.000 f$ \\
\hline & $G^{3}(4 \mathrm{f}, 6 \mathrm{~s})$ & 2749 & 2083 & 0.758 \\
\hline \multirow[t]{2}{*}{$4 f^{7} 5 d-4 f^{7} 6 s$} & $R^{2}(4 \mathrm{f}, 5 \mathrm{~d} ; 4 \mathrm{f}, 6 \mathrm{~s})$ & -214 & -182 & $0.850 f$ \\
\hline & $R^{2}(4 \mathrm{f}, 5 \mathrm{~d} ; 4 \mathrm{f}, 6 \mathrm{~s})$ & 2162 & 1838 & $0.850 f$ \\
\hline \multicolumn{5}{|l|}{ Odd parity } \\
\hline \multirow[t]{9}{*}{$4 \mathrm{f}^{7} 6 \mathrm{p}$} & $E_{\mathrm{av}}$ & 139046 & 138527 & \\
\hline & $F^{2}(4 \mathrm{f}, 4 \mathrm{f})$ & 116761 & 99247 & $0.850 f$ \\
\hline & $F^{4}(4 \mathrm{f}, 4 \mathrm{f})$ & 73267 & 62277 & $0.850 f$ \\
\hline & $F^{6}(4 \mathrm{f}, 4 \mathrm{f})$ & 52712 & 44805 & $0.850 f$ \\
\hline & $\zeta_{4 \mathrm{f}}$ & 1589 & 1589 & $1.000 f$ \\
\hline & $\zeta_{6 p}$ & 2469 & 3026 & 1.226 \\
\hline & $F^{2}(4 \mathrm{f}, 6 \mathrm{p})$ & 7447 & 6330 & $0.850 f$ \\
\hline & $G^{2}(4 \mathrm{f}, 6 \mathrm{p})$ & 1881 & 1762 & $0.937 *$ \\
\hline & $G^{4}(4 \mathrm{f}, 6 \mathrm{p})$ & 1689 & 1582 & $0.937 *$ \\
\hline \multirow[t]{5}{*}{$4 f^{8}$} & $E_{\mathrm{av}}$ & 84926 & 65548 & \\
\hline & $F^{2}(4 \mathrm{f}, 4 \mathrm{f})$ & 108539 & 92258 & $0.850 f$ \\
\hline & $F^{4}(4 \mathrm{f}, 4 \mathrm{f})$ & 67727 & 57568 & $0.850 f$ \\
\hline & $F^{6}(4 \mathrm{f}, 4 \mathrm{f})$ & 48620 & 41327 & $0.850 f$ \\
\hline & $\zeta_{4 \mathrm{f}}$ & 1466 & 1359 & 0.927 \\
\hline \multirow[t]{2}{*}{$4 f^{7} 6 p-4 f^{8}$} & $R^{2}(4 \mathrm{f}, 6 \mathrm{p} ; 4 \mathrm{f}, 4 \mathrm{f})$ & -4099 & -3484 & $0.850 f$ \\
\hline & $R^{4}(4 \mathrm{f}, 6 \mathrm{p} ; 4 \mathrm{f}, 4 \mathrm{f})$ & -2509 & -2133 & $0.850 f$ \\
\hline
\end{tabular}

$f$ Fixed parameter (see the text).

* Fixed ratio (see the text).

observations due to Callahan (1963), Johansson \& Litzén (1973), Kielkopf (1976) and Wyart (1976). More precisely, from his analyis of the $4 \mathrm{f}^{7}\left({ }^{8} \mathrm{~S}^{\circ}\right) 5 \mathrm{~d}-6 \mathrm{p}$ and $4 \mathrm{f}^{7}\left({ }^{8} \mathrm{~S}^{\circ}\right) 6 \mathrm{~s}-6 \mathrm{p}$ transitions in the region 2043-3177 $\AA$, Callahan (1963) was able to classify 42 lines and to deduce the $4 \mathrm{f}^{7} 5 \mathrm{~d}$, $6 \mathrm{~s}$ and $6 \mathrm{p} \mathrm{lev-}$ els. The five known levels of the $4 \mathrm{f}^{8}{ }^{7} \mathrm{~F}$ term were obtained by Johansson \& Litzén (1973) from the ${ }^{7} \mathrm{~F}-{ }^{7} \mathrm{D}^{\circ}$ multiplet observed in the infrared region $(1.43-2.56 \mu \mathrm{m})$. Kielkopf (1976) has reevaluated the levels on the basis of his own observations and his levels were adopted by Martin et al. (1978) with the exception of the two $4 \mathrm{f}^{7}\left({ }^{8} \mathrm{~S}^{\circ}\right) 7 \mathrm{~s}$ levels taken from Wyart (1976).

Concerning the transition rates, neither theoretical nor experimental transition probabilities have been determined so far. Natural radiative lifetimes of five $4 f^{7} 6 p$ levels in Gd III
Table 2. Comparison between calculated radiative lifetimes, $\tau$ in ns, obtained in the present work without $(H F R)$ and with $(H F R+C P)$ core-polarization contributions and experimental results for $4 \mathrm{f}^{7} 6 \mathrm{p} \mathrm{lev-}$ els of Gd III.

\begin{tabular}{ccccc}
\hline \hline$E\left(\mathrm{~cm}^{-1}\right)$ & $J$ & $H F R$ & $H F R+C P$ & Experiment* \\
\hline 43019.99 & 3 & 1.41 & 1.87 & $1.9 \pm 0.2$ \\
43611.69 & 4 & 1.49 & 1.97 & $1.9 \pm 0.2$ \\
47233.93 & 5 & 1.06 & 1.41 & $1.4 \pm 0.2$ \\
48339.14 & 4 & 1.24 & 1.65 & $1.5 \pm 0.2$ \\
48859.62 & 3 & 1.32 & 1.75 & $1.5 \pm 0.2$ \\
\hline
\end{tabular}

* Zhang et al. (2001b).

were measured for the first time by Zhang et al. (2001b) using time-resolved laser-induced fluorescence in a laser-produced plasma. No additional results were available for comparison.

\section{Calculations and discussion of the results}

In the present work, we report on the first theoretical investigation of transition rates in Gd III. The new results were calculated in the framework of the relativistic Hartree-Fock (HFR) method coded in Cowan's (1981) suite of computer programs in which we have incorporated the core-polarization (CP) effects by means of a model potential and a correction to the electric dipole operator. The method is similar to that previously described (see e.g. Biémont et al. 2001a,b,c,d) and, consequently, the details will not be repeated here. The configurations retained in the physical model were $4 f^{7} 5 d, 4 f^{7} 6 s$ for the odd parity and $4 \mathrm{f}^{7} 6 \mathrm{p}, 4 \mathrm{f}^{8}$ for the even parity. This model is clearly simple but the consideration of additional configurations was prevented by the computer capabilities available. However, the lifetime values deduced in the present work (see further) justify a posteriori the model used. The core-polarization contributions were estimated using the static dipole polarizability computed by Fraga et al. (1976) for Gd IV, i.e. $\alpha_{\mathrm{d}}=6.81 \mathrm{a}_{0}^{3}$, and the cut-off radius, $r_{\mathrm{c}}=1.55 \mathrm{a}_{0}$, which corresponds to the average $H F R$ value $\langle r\rangle$ for the outermost core orbital $\left(5 \mathrm{p}^{6}\right)$. A least-squares optimization procedure of the radial parameters was followed in order to minimize the differences between the calculated levels and the experimental values compiled by the NIST (Martin et al. 1978). In view of the scarcity of these experimental levels, we were able to adjust only a few parameters. More precisely, since only the ${ }^{8} \mathrm{~S}^{\circ}$ parent term is known in the $4 \mathrm{f}^{7} n l(n l=5 \mathrm{~d}, 6 \mathrm{~s}, 6 \mathrm{p})$ configurations, only the parameters $E_{\mathrm{av}}$, $\zeta_{n l}$ and $G^{k}(4 \mathrm{f}, n l)$ were allowed to vary with the constraint that the ratios $G^{1} / G^{3} / G^{5}$ and $G^{2} / G^{4}$ were kept fixed during the fitting process concerning the $4 \mathrm{f}^{7} 5 \mathrm{~d}$ and $4 \mathrm{f}^{7} 6 \mathrm{p}$ configurations, respectively. For $4 \mathrm{f}^{8}$, only $E_{\text {av }}$ and $\zeta_{4 \mathrm{f}}$ could be adjusted due to the fact that only the ${ }^{7} \mathrm{~F}$ multiplet is determined experimentally in this configuration. All the other electrostatic parameters, not optimized semi-empirically, were scaled down by a factor equal to 0.85 . The mean deviations between fitted and observed levels in the odd and even parities were found to be equal to $25 \mathrm{~cm}^{-1}$ (12 levels) and $8 \mathrm{~cm}^{-1}$ (11 levels), respectively. The numerical values of the radial parameters adopted in the HFR calculations are reported in Table 1. 
Table 3. Calculated oscillator strengths $(\log g f)$ and transition probabilities $(g A)$ for $4 \mathrm{f}^{7} 5 \mathrm{~d}-4 \mathrm{f}^{7} 6 \mathrm{p}$ and $4 \mathrm{f}^{7} 6 \mathrm{~s}-4 \mathrm{f}^{7} 6 \mathrm{p}$ transitions in Gd III. $A(+B)$ stands for $A \times 10^{B}$.

\begin{tabular}{|c|c|c|c|c|c|c|c|c|}
\hline \multirow[t]{2}{*}{$\lambda(\AA)^{a}$} & \multicolumn{3}{|c|}{ Lower level } & \multicolumn{3}{|c|}{ Upper level } & \multirow[t]{2}{*}{$\log g f$} & \multirow[t]{2}{*}{$g A\left(s^{-1}\right)$} \\
\hline & Config. & $J$ & $E\left(\mathrm{~cm}^{-1}\right)$ & Config. & $J$ & $E\left(\mathrm{~cm}^{-1}\right)$ & & \\
\hline $2032.085^{b}$ & $4 f^{7} 5 d$ & 2.0 & 0 & $41^{\circ}$ op & 2.0 & 49195 & -2.36 & $6.98(+6)$ \\
\hline 2043.698 & $4 f^{7} 5 d$ & 3.0 & 279 & $4 f^{7} 6 p$ & 2.0 & 49195 & -2.21 & $9.81(+6)$ \\
\hline 2046.029 & $4 f^{7} 5 d$ & 2.0 & 0 & $4 f^{7} 6 p$ & 3.0 & 48860 & -1.06 & $1.38(+8)$ \\
\hline 2057.792 & $4 f^{7} 5 d$ & 3.0 & 279 & $4 f^{7} 6 p$ & 3.0 & 48860 & -1.37 & $6.69(+7)$ \\
\hline $2075.524^{b}$ & $4 f^{7} 5 d$ & 4.0 & 694 & $4 f^{7} 6 p$ & 3.0 & & -2.29 & $7.96(+6)^{*}$ \\
\hline 2080.080 & $4 f^{7} 5 d$ & 3.0 & 279 & $4 f^{7} 6 p$ & 4.0 & 48339 & -0.88 & $2.05(+8)$ \\
\hline 2098.204 & $4 f^{7} 5 d$ & 4.0 & 694 & $4 f^{7} 6 p$ & 4.0 & 48339 & -0.63 & $3.57(+8)$ \\
\hline 2125.683 & $4 f^{7} 5 d$ & 5.0 & 1310 & $4 f^{7} 6 p$ & 4.0 & 48339 & -0.74 & $2.72(+8)$ \\
\hline 2148.034 & $4 \mathrm{f}^{7} 5 \mathrm{~d}$ & 4.0 & 694 & $4 f^{7} 6 p$ & 5.0 & 47234 & -0.83 & $2.13(+8)$ \\
\hline 2176.844 & $4 f^{7} 5 d$ & 5.0 & 1310 & $4 f^{7} 6 p$ & 5.0 & & 0.17 & \\
\hline 2223.943 & $4 f^{7} 5 d$ & 6.0 & 2283 & $4 f^{7} 6 p$ & 5.0 & & 0.31 & \\
\hline 2307.031 & $4 f^{7} 5 d$ & 3.0 & 279 & $4 f^{7} 6 p$ & 4.0 & 43612 & -0.57 & $3.38(+8)$ \\
\hline 2323.783 & $4 f^{7} 5 d$ & 2.0 & 0 & $4 f^{7} 6 p$ & 3.0 & 43020 & -0.17 & $8.27(+8)$ \\
\hline 2329.347 & $4 f^{7} 5 d$ & 4.0 & 694 & $4 f^{7} 6 p$ & 4.0 & & -0.20 & \\
\hline 2338 & $4 f^{7} 5 d$ & 3.0 & 279 & $4 f^{7} 6 p$ & 3.0 & & -0.21 & \\
\hline 2361 & $4 f^{7} 5 d$ & 4.0 & 694 & $4 f^{7} 6 p$ & 3.0 & & -0.46 & $4.17(+8)$ \\
\hline 2363.261 & $4 f^{7} 5 d$ & 5.0 & 1310 & $4 f^{7} 6 p$ & 4.0 & 43 & -0.08 & $9.86(+8)$ \\
\hline 2520.389 & $4 f^{7} 6 s$ & 4.0 & 9195 & $4 f^{7} 6 p$ & 3.0 & 48 & -0.45 & $3.73(+8)$ \\
\hline 2551.560 & $4 f^{7} 5 d$ & 3.0 & 10015 & $4 f^{7} 6 p$ & 2.0 & & -0.58 & \\
\hline 255 & $4 f^{7} 6 s$ & 4.0 & 9195 & $4 f^{7} 6 p$ & 4.0 & & 0.09 & $+9)$ \\
\hline 2554.045 & $4 f^{7} 5 d$ & 4.0 & 9718 & $4 f^{7} 6 p$ & 3.0 & 48860 & -0.17 & $6.93(+8)$ \\
\hline 2564.469 & $4 f^{7} 5 d$ & 5.0 & 9356 & $4 f^{7} 6 p$ & 4.0 & 48339 & 0.04 & $1.12(+9)$ \\
\hline 2565.9 & $4 f^{7} 5 d$ & 2.0 & 10234 & $4 f^{7} 6 p$ & 2.0 & & -0.36 & \\
\hline & $4 f^{7} 5 d$ & 3.0 & 15 & $4 f^{7} 6 p$ & 3.0 & & -0.27 & \\
\hline 2576.068 & $4 f^{7} 5 d$ & 1.0 & 10387 & $4 f^{7} 6 p$ & 2.0 & 49 & -0.40 & $3.99(+8)$ \\
\hline 2588.221 & $4 f^{7} 5 d$ & 2.0 & 10234 & $4 f^{7} 6 p$ & 3.0 & 48 & -0.68 & $2.08(+8)$ \\
\hline 2588.467 & $4 f^{7} 5 d$ & 4.0 & 9718 & $4 f^{7} 6 p$ & 4.0 & & -0.43 & $3.74(+8)$ \\
\hline & $4 f^{7} 5 d$ & 3.0 & 10015 & $4 f^{7} 6 p$ & 4.0 & & -1.13 & 7.3 \\
\hline 262 & $4 f^{7} 6 s$ & 4.0 & 9195 & $4 f^{7} 6 p$ & 5.0 & 47 & 0.60 & $3.86(+9)$ \\
\hline 2639.299 & $4 f^{7} 5 d$ & 5.0 & 9356 & $4 f^{7} 6 p$ & 5.0 & 47 & -1.72 & $1.85(+7)$ \\
\hline 2655.599 & $4 f^{7} 6 s$ & 3.0 & 11550 & $4 f^{7} 6 p$ & 2.0 & 49195 & 0.25 & $1.70(+9)$ \\
\hline 2664.722 & $4 f^{7} 5 d$ & 4.0 & 9718 & $4 f^{7} 6 p$ & 5.0 & 47234 & -2.43 & $3.48(+6)$ \\
\hline 2679.449 & $4 \mathrm{f}^{7} 6 \mathrm{~s}$ & 3.0 & 11550 & $4 f^{7} 6 p$ & 3.0 & & 0.33 & $1.97(+9)$ \\
\hline 2717.357 & $4 f^{7} 6 s$ & 3.0 & 11550 & $4 f^{7} 6 p$ & 4.0 & 48 & 0.30 & $1.80(+9)$ \\
\hline 2904.726 & $4 f^{7} 6 s$ & 4.0 & 9195 & $4 f^{7} 6 p$ & 4.0 & 43612 & 0.27 & $1.46(+9)$ \\
\hline 2918.398 & $4 f^{7} 5 d$ & 5.0 & 9356 & $4 f^{7} 6 p$ & 4.0 & 43612 & -0.52 & $2.35(+8)$ \\
\hline 2949.512 & $4 f^{7} 5 d$ & 4.0 & 9718 & $4 f^{7} 6 p$ & 4.0 & 43612 & -1.17 & $5.24(+7)$ \\
\hline 2955.534 & $4 f^{7} 6 s$ & 4.0 & 9195 & $4 f^{7} 6 p$ & 3.0 & 43020 & 0.29 & $1.49(+9)$ \\
\hline 2975.594 & $4 f^{7} 5 d$ & 3.0 & 10015 & $4 f^{7} 6 p$ & 4.0 & 43612 & -1.99 & $7.75(+6)$ \\
\hline 3001.918 & $4 f^{7} 5 d$ & 4.0 & 9718 & $4 f^{7} 6 p$ & 3.0 & 43020 & -1.36 & $3.21(+7)$ \\
\hline 3028.936 & $4 f^{7} 5 d$ & 3.0 & 10015 & $4 f^{7} 6 p$ & 3.0 & 43020 & -1.55 & $2.05(+7)$ \\
\hline 3049.241 & $4 f^{7} 5 d$ & 2.0 & 10234 & $4 f^{7} 6 p$ & 3.0 & 43020 & -2.02 & $6.81(+6)$ \\
\hline 3118.041 & $4 f^{7} 6 s$ & 3.0 & 11550 & $4 f^{7} 6 p$ & 4.0 & 43612 & 0.00 & $6.90(+8)$ \\
\hline 3176.662 & $4 f^{7} 6 s$ & 3.0 & 11550 & $4 f^{7} 6 p$ & 3.0 & 43020 & -0.54 & $1.90(+8)$ \\
\hline
\end{tabular}

${ }^{a}$ From Callahan (1963) except when otherwise indicated.

${ }^{b}$ Deduced from the experimental energy levels (Martin et al. 1978).

* Transition probability possibly affected by cancellation effects.

The radiative lifetimes calculated in the present work, without $(H F R)$ and with $(H F R+C P)$ core-polarization corrections and with the fitted parameters, are compared in Table 2 with the available (uncertainties of about 10-15\%) laser measurements of Zhang et al. (2001b) published for five $4 \mathrm{f}^{7} 6 \mathrm{p}$ levels of Gd III. As seen from this table, the agreement between our $H F R+C P$ results and the experimental values is excellent, the mean ratio $\tau_{\exp } / \tau_{H F R+C P}$ being equal to $0.95 \pm 0.06$ where the uncertainty represents the standard deviation of the mean.

Calculated oscillator strengths and transition probabilities are reported in Table 3 for $444 \mathrm{f}^{7} 5 \mathrm{~d}-6 \mathrm{p}$ and $4 \mathrm{f}^{7} 6 \mathrm{~s}-6 \mathrm{p}$ transitions in the spectral range $2000-3200 \AA$ A. In view of the close 
Table 4. Calculated Lande factors for low-lying levels of Gd III.

\begin{tabular}{rlc}
\hline \hline$E\left(\mathrm{~cm}^{-1}\right)$ & $J$ & Lande factor \\
\hline 0.00 & 2 & 2.653 \\
279.32 & 3 & 2.071 \\
694.37 & 4 & 1.839 \\
1310.13 & 5 & 1.725 \\
2282.83 & 6 & 1.663 \\
2381.24 & 6 & 1.495 \\
3996.71 & 5 & 1.496 \\
5015.20 & 4 & 1.497 \\
5789.97 & 3 & 1.497 \\
6334.17 & 2 & 1.498 \\
9195.04 & 4 & 1.995 \\
9356.30 & 5 & 1.600 \\
9717.70 & 4 & 1.651 \\
10014.75 & 3 & 1.750 \\
10234.49 & 2 & 1.997 \\
10387.30 & 1 & 2.984 \\
11549.50 & 3 & 1.992 \\
43019.99 & 3 & 2.196 \\
43611.69 & 4 & 1.863 \\
47233.93 & 5 & 1.796 \\
48339.14 & 4 & 1.819 \\
48859.62 & 3 & 1.935 \\
49194.70 & 2 & 2.321 \\
\hline
\end{tabular}

agreement between theoretical and observed lifetime values, the theoretical transition probabilities were not renormalized with the experimental data. Such a procedure would change the final data by only a few $\%$ and could be easily applied if needed. It is clear also that the agreement between the theoretical and experimental lifetime values is a necessary but not sufficient condition to definitely assess the accuracy of the final transition probabilities. Measurement of accurate branching fractions would be necessary as an additional step but such measurements have not been performed so far. However, in view of the accuracy reached in other doubly ionized elements of the same group (see the DREAM database and the relevant publications) and of the fact that most of the transitions considered in the present paper are rather strong, uncertainties of the order of 10-20\% are probably to be expected for most of the transitions quoted in Table 3. Only one transition of Table 3 ( 22075.524$)$ is likely to be affected by cancellation effects.

In view of their intrinsic interest for the analysis of the spectra of magnetic stars, we report in Table 4 the calculated Lande factors for 23 low-lying levels of Gd III.

\section{Conclusions}

A first set of transition probabilities has been obtained in Gd III for $444 f^{7} 5 d-6 p$ and $4 f^{7} 6 s-6 p$ transitions of astrophysical interest appearing in the spectral range 2000-3200 $\AA$. These results will help provide a quantitative analysis of UV spectra of chemically peculiar stars and will allow the astrophysicists to deduce the chemical composition of some stars from the lines emitted by the dominant (doubly ionized) species of this atom.
Due to the lack of experimental energy levels in Gd III, the set of oscillator strengths reported in the present paper is limited to lines appearing in the near ultraviolet region, which means that they are restricted to observations made from space. In order to extend the study of radiative properties in the visible range for the interpretation of ground-based spectra, new term analysis of this ion would be very welcome.

Acknowledgements. Financial support from the Belgian National Fund for Scientific Research (FNRS) is acknowledged.

\section{References}

Adelman, S. J. 1989, MNRAS, 239, 487

Anders, E., \& Grevesse, N. 1989, Geochim. Cosmochim. Acta, 53, 197

Bergström, H., Biémont, E., Lundberg, H., \& Persson, A. 1988, A\&A, 192, 335

Bidelman, W. P., Cowley, C. R., \& Iler, A. L. 1995, Pub. Obs. Univ. Mich., 12, 3

Biémont, E., Li, Z. S., Palmeri P., \& Quinet, P. 1999, J. Phys. B, 32, 3409

Biémont, E., Garnir, H. P., Bastin, T., et al. 2001a, MNRAS, 321, 481

Biémont, E., Garnir, H. P., Palmeri, P., et al. 2001b, Phys. Rev. A, 64, 022503

Biémont, E., Garnir, H. P., Li, Z. S., et al. 2001c, J. Phys. B, 34, 1869

Biémont, E., Palmeri, P., Quinet, P., et al. 2001d, MNRAS, 328, 1085

Biémont, E., Quinet, P., \& Ryabchikova, T. A. 2002, in preparation

Callahan, W. R. J. 1963, JOSA, 53, 695

Cohen, J. G., Deutsch, A. J., \& Greenstein, J. L. 1969, ApJ, 156, 629

Cowan, R. D. 1981, The Theory of Atomic Structure and Spectra (Univ. California Press, Berkeley)

Cowley, C. R. 1980, Vistas Astron., 24, 245

Cowley, C. R., \& Greenberg, M. 1988, MNRAS, 232, 763

Cowley, C. R., Ryabchikova, T., Kupka, F., et al. 2000, MNRAS, 317, 299

Fedchak J. A., Den Hartog, E. A., Lawler, J. E., et al. 2000, ApJ, 542, 1109

Fraga, S., Karwowski, J., \& Saxena, K. M. S. 1976, Handbook of Atomic Data (Elsevier, Amsterdam)

Grudzinska, S. 1985, in Cool Stars with Excesses of Heavy Elements, ed. M. Jaschek, \& P. C. Keenan (Reidel), p. 267

Hartoog, M. R., Cowley, C. R., \& Cowley, A. P. 1973, ApJ, 182, 847

Johansson, S., \& Litzén, U. 1973, Phys. Scr., 8, 43

Kielkopf, J. 1976, unpublished material

Lambert, D. L. 1985, in Cool Stars with Excesses of Heavy Elements, ed. M. Jaschek, \& P. C. Keenan (Reidel), p. 191

Li, Z. S., Zhang, Z. G., Lokhnygin, V., et al. 2001, J. Phys. B, 34, 1349

Magazzu, A., \& Cowley, C. R. 1986, ApJ, 308, 254

Martin, W. C., Zalubas, R., \& Hagan, L. 1978, Atomic Energy Levels - The Rare-Earth Elements, NSRDS-NBS (U.S.) 60

Palmeri, P., Quinet, P., Frémat, Y., Wyart, J.-F., \& Biémont, E. 2000, ApJS, 129, 367

Sneden, C., McWilliam, A., Preston, G. W., et al. 1996, ApJ, 467, 819

Wyart, J.-F. 1976, unpublished material

Zhang, Z. G., Li, Z. S., Svanberg, S., et al. 2001a, Eur. Phys. J. D, 15, 301

Zhang, Z. G., Persson, A., Li, Z. S., Svanberg, S., \& Jiang Zhankui, 2001b, Eur. Phys. J. D, 13, 301

Zhang, Z. G., Somesfalean, G., Svanberg, S., et al. 2002a, A\&A, 384, 364

Zhang, Z. G., Svanberg, S., Palmeri, P., Quinet, P., \& Biémont, E. 2002b, A\&A, 385, 724

Zhang, Z. G., Svanberg, S., Palmeri, P., Quinet, P., \& Biémont, E. 2002c, MNRAS, in press 